\title{
Review: antidepressants improve symptoms of fibromyalgia
}

O'Malley PG, Balden E, Tomkins G, et al. Treatment of fibromyalgia with antidepressants. A meta-analysis. J Gen Intern Med 2000 Sep;15:659-66.

\section{QUESTION: Are antidepressants efficacious in the treatment of fibromyalgia?}

\section{Data sources}

Studies in any language were identified by searching Medline (1966 to June 1999), PsycLIT (1974-98), and EMBASE/Excerpta Medica (1974-98) with the terms fibromyalgia, fibrositis, and fibromyositis combined with various terms for antidepressive agents (including specific drug names); the Cochrane Library Clinical Trials Registry and Database of Systematic Reviews; Federal Research in Progress to identify unpublished trials; and bibliographies of reviewed articles.

\section{Study selection}

Studies were selected if they were randomised, placebo controlled trials with at least 1 group receiving an antidepressant and if they reported measurable outcomes.

\section{Data extraction}

Data were extracted on setting; country; dose, duration, and follow up of treatment; number of participants and demographics; assessment of comorbid disease; follow up losses; adverse effects; and outcomes. Methodological quality of individual trials was assessed using the Jadad scale.

\section{Main results}

13 trials (mean sample size 54, mean duration 8.1 wks) were included in the analysis. Mean quality score for the Jadad scale was 5.6 out of 8 . Nine studies did not state the method of randomisation, 7 had no intention to treat analysis, 3 had inadequate assurance of blinding effectiveness, 5 had no assessment of adverse effects, and 6 had $>20 \%$ loss to follow up. Trials assessed the efficacy of 3 classes of antidepressants: tricyclics (8 trials of amitriptyline, 1 of clomipramine, and 1 of maprotiline); S-adenosylmethionine (2 trials); and selective serotonin reuptake inhibitors (2 of fluoxetine and 1 of citalopram). Meta-analysis was done using a random effects model. Meta-analysis of dichotomous outcome data (10 trials) showed that patients who received antidepressants had more symptom improvement than those who received placebo (odds ratio 4.2, 95\% CI 2.6 to 6.8 ; number needed to treat 4 , CI 2.9 to 6.3 ). Meta-analysis of continuous outcome data showed that antidepressants were associated with improvements in fatigue scores (8 trials, $0.39 \mathrm{SD}$ unit improvement, CI 0.11 to 0.66 ), sleep scores ( 8 trials, $0.49 \mathrm{SD}$ unit improvement, CI 0.3 to 0.69 ), pain scores (10 trials, 0.52 SD unit improvement, CI 0.21 to 0.81 ), and overall wellbeing ( 7 trials, $0.49 \mathrm{SD}$ unit improvement, CI 0.18 to 0.80 ) but not in the number of trigger points.

\section{Conclusion}

Antidepressants improve overall symptoms and individual symptoms of fatigue, sleep, and pain in fibromyalgia.

\section{COMMENTARY}

O'Malley et al did a systematic review of 13 randomised, placebo controlled trials that included $\geqslant 1$ medication arm with an antidepressant, usually a tricyclic. Analysis showed heterogeneous effect sizes for pain, wellbeing, sleep, fatigue, and number of trigger points (also called tender points). Summary effect sizes of the 4 self reported measurements were statistically significant and showed a moderate treatment effect. The physician reported number of tender points did not differ by treatment group. As the authors state, however, adequate blinding in trials was difficult because of the side effects of antidepressants. Moreover, the authors found statistical evidence for publication bias.

The results from the 13 articles were probably analysed by dichotomous classification in favour of the antidepressant or placebo, a classification based on statistical rather than clinical relevance. The authors presented the results in terms of odds ratios and numbers needed to treat (NNTs). This representation of results is difficult to interpret.

The authors conclude that antidepressants are effective for fibromyalgia. This conclusion appears too strong from both the methodological and the clinical perspectives. Heterogeneity of results between (and undoubtedly also within) studies suggests that antidepressants are moderately effective in only some patients. Another meta-analysis of 49 studies assessed the efficacy of pharmacological and non-pharmacological interventions for fibromyalgia. ${ }^{1}$ In this review, exercise and cognitive behavioural therapy were more efficacious than pharmacological treatment alone for self reported fibromyalgia symptoms. A similar trend was observed for functional measurements. These findings suggest that the treatment of choice in fibromyalgia is daily physical exercise and encouragement of positive behaviour Rather than analysing the effects of pharmacological treatments per se, the objective of future evaluations should be to determine which patients profit from antidepressants as adjuncts to non-pharmacological interventions and which antidepressants perform best.

Johannes W G Jacobs, MD, PhD

R Geenen, $\mathrm{PhD}$ University Medical Centre Utrecht, the Netherlands

1 Rossy LA, Buckelew SP, Dorr N, et al. A meta-analysis of fibromyalgia treatment interventions. Ann Behav Med 1999;21:180-91.

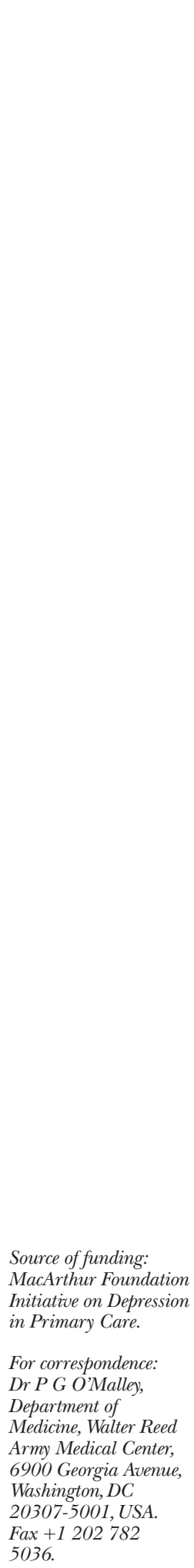

